\title{
Elucidating chromatin and nuclear domain architecture with electron spectroscopic imaging
}

\author{
David P. Bazett-Jones ${ }^{1 *}$, Ren $\mathrm{Li}^{1}$, Eden Fussner ${ }^{1}$, Rosa Nisman ${ }^{1} \&$ Hesam Dehghani ${ }^{2}$ \\ ${ }^{1}$ Program in Genetics and Genome Biology, The Hospital for Sick Children, Research Institute, 101 College \\ Street, East Tower, 15th Floor, 15-401T, Toronto, ON M5G 1L7, Canada; Tel: +1-416-813-2181; E-mail: \\ dbjones@sickkids.ca; ${ }^{2}$ Department of Physiology, School of Veterinary Medicine, \\ Ferdowsi University of Mashhad, Mashhad, Iran \\ The authors dedicate this paper to the memory of Ying Ren (1961-2007). We all benefited from knowing her. Our \\ research advanced through the technical creativity she provided. \\ * Correspondence
}

Key words: chromatin, correlative microscopy, electron microscopy, heterochromatin, nuclear speckles, nuclear structure, nucleolus, promyelocytic leukemia, transcription

\begin{abstract}
Electron microscopy has been the 'gold standard' of spatial resolution for studying the structure of the cell nucleus. Electron spectroscopic imaging (ESI) offers advantages over conventional transmission electron microscopy by eliminating the need for heavy-atom contrast agents. ESI also provides mass-dependent and element-specific information at high resolution, permitting the distinguishing of structures that are primarily composed of protein, DNA, or RNA. The technique can be applied to understand the structural consequences of epigenetic modifications, such as modified histones, on chromatin fiber morphology. ESI can also be applied to elucidate the multifunctional behavior of subnuclear 'organelles' such as the nucleolus and promyelocytic leukemia nuclear bodies.

$\begin{array}{ll}\text { Abbreviations } \\ \text { CBP } & \begin{array}{l}\text { Creb-binding protein } \\ \text { condensed chromatin } \\ \text { conventional transmission electron microscopy } \\ \text { CC }\end{array} \\ \text { CTEM } & \text { murine embryonic stem cell } \\ \text { Daxx } & \text { electron spectroscopic imaging } \\ \text { ES } & \text { green fluorescent protein } \\ \text { ESI } & \text { interchromosome space } \\ \text { GFP } & \text { interchromatin granule clusters } \\ \text { ICS } & \text { inhibitor of growth family member 1 } \\ \text { IGC } & \text { murine erythroleukemia cell } \\ \text { ING1 } & \text { murine double minute protein 2 } \\ \text { MEL } & \text { promyelocytic leukemia nuclear body } \\ \text { Mdm2 } & \text { retinoblastoma } \\ \text { PML NB } & \end{array}$

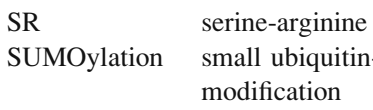

\section{Introduction}

The cell nucleus is composed of several compartments including chromosome territories, nucleoli, nuclear speckles (interchromatin granule clusters), replication and transcription factories, promyelocytic leukemia nuclear bodies (PML NBs), and a number of other bodies and assemblies. All await further analysis and functional characterization. How these structures within the nucleus are organized appears to
\end{abstract}


be largely a function of the various nuclear activities, such as transcription or replication of DNA (Kosak \& Groudine 2004), yet the fundamental rules governing the relationship of nuclear structure to function remain to be elucidated.

Nuclear compartments are assembled and maintained in a dynamic fashion (Misteli 2001), likely dictated by their function and involvement in nuclear processes. Nucleolar organization, for example, provides a unique spatial clustering of ribosomal genes from different chromosomes to a defined nuclear volume that enriches all of the players involved in transcription of ribosomal RNA genes. In another example, accumulation of a set of active or potentially active genes likely contributes to the assembly of 'transcription factories', structures that provide a local permissive microenvironment for regulation and activity of RNA polymerase II (Osborne et al. 2004). Our understanding of the relationships of chromatin, gene activation and subnuclear domains has progressed with technical developments in cell imaging. Conventional transmission electron microscopy (CTEM), for example, has revealed dense packing of chromatin, thought to be heterochromatin, along the nuclear envelope and at the periphery of the nucleolus. In parallel, advances in light microscopy over the past two decades have revealed other structural features such as the territorial organization of chromosomes and dynamic features of both nuclear bodies and specific chromosome loci. However, the temptation to infer molecular detail from typical light microscopy observations, limited to $200 \mathrm{~nm}$, can lead to over-interpretation of data, which in turn leads to erroneous conclusions about nuclear structure and function (Dehghani et al. 2005). Efforts are now underway to fill this resolution gap between the conventional light microscope and the electron microscope.

\section{Electron spectroscopic imaging}

Electron spectroscopic imaging (ESI) has helped us to address several diverse questions in both molecular and cellular biology (Bazett-Jones \& Hendzel 1999, Bazett-Jones et al. 1999, Dellaire \& BazettJones 2004). The technique is based on the principle that some incident electrons that pass through a specimen in the transmission electron microscope will lose energy due to ionization of both valence shell and core electrons of the specimen's atoms. The energy losses that arise from core loss ionizations are discrete and are element-specific. The incident electrons, which cause these events, could therefore be used to generate element-specific images if it were possible to filter or select these electrons from the electron energy loss spectrum. Electron imaging spectrometers have been designed and implemented for this purpose. These devices accomplish two functions. First, in functioning as an electron spectrometer, they separate electrons according to their energy losses. This creates an electron energy loss spectrum. Their second function is to remove aberrations created by the spectrum-forming operation, and to create an aberration-corrected image of the specimen. The two functions together create an aberration-corrected element-specific image.

Electron energy loss imaging, which we call electron spectroscopic imaging (ESI) offers advantages over CTEM. First, the removal of electrons that do not interact with the specimen as well as the selection of electrons that have undergone a specific energy loss through specimen interactions produces a dark-field image. Such images can provide a great increase in contrast over a bright-field CTEM image. Whereas heavy-atom contrast agents, such as uranium and lead salts, are required to provide sufficient contrast of poorly scattering light elements, i.e. those elements that are relevant to biological material, ESI does not require these contrast-enhancing stains. This is important for two reasons. The contrast agents function by coating the biological material with large precipitates of stain, consequently limiting the spatial resolution. Second, these heavy atom salts do not interact uniformly with each biochemical component; some compounds bind large amounts of uranium acetate, for example, whereas other biochemicals do not interact at all with the salt. This non-uniformity can create false impressions of 'electron-dense' regions, and mass-depleted regions. In fact, the true mass density may not be reflected accurately by the uranium salt interactions.

A second advantage of ESI is that element-specific maps can be created. This opportunity can be exploited in cell-free studies of purified ribonucleoprotein (RNP) structures or DNA:protein complexes. The nucleic acid component contains significantly more phosphorus than the protein component of such complexes. Phosphorus maps, therefore, provide a means of distinguishing the nucleic acid distribution in such complexes. Moreover, quantitative evaluation 
of these phosphorus-specific maps provides a measure for nucleic acid in the complex. Mass-sensitive images, recorded in regions of the energy loss spectrum that do not correspond to phosphorus, provide total mass information. Comparisons of the phosphorus maps and the mass images can therefore be used to calculate stoichiometric relationships between the nucleic acid and protein components of the complex. For these reasons, ESI has been applied to the study of a variety of DNA: protein structures that are relevant to gene regulation. For example, important architectural features of transcription factor interactions with promoter elements have been observed (Bazett-Jones et al. 1994, Brown et al. 1996, Stefanovsky et al. 2001). At the level of chromatin organization, the importance of specific chromatin modifications that destabilize nucleosome structure (Bazett-Jones \& Hendzel 1999), and how a nucleosome remodeling complex can provide access to DNA in the nucleosome (Bazett-Jones et al. 1996) have been described. In addition, ESI has provided the information to suggest a mechanism by which condensins complex DNA in metaphase chromosomes (Bazett-Jones et al. 2002).

In this review, we describe how phosphorus and nitrogen mapping can be applied to studies of intact nuclei, to address questions that are relevant to nuclear functions (Bazett-Jones \& Hendzel 1999, Hendzel et al. 1999, Dellaire et al. 2004). An important technical advance has been to correlate fluorescence microscopy and ESI on the same serial sections. This provides a method to determine the ultrastructural basis for an observation obtained by fluorescence microscopy (Ren et al. 2003, Dellaire \& Bazett-Jones 2004).

\section{High-resolution biochemical detail revealed by phosphorus and nitrogen mapping}

The contrast provided by ESI by energy loss darkfield and through element-specific contrast permits the imaging of biological material without heavyatom contrast agents. As a result, the biological material itself, rather than a heavy-atom coating of the biomaterial can be imaged. Additionally, elementspecific information obtained at a high degree of spatial resolution permits the delineation of nucleic acid and protein. This is illustrated in energy-filtered images of a cell from a 16-cell pre-implantation mouse embryo (Figure 1). To prepare the sample for electron microscopy, the embryo was removed from the mother and fixed for $30 \mathrm{~min}$ in $4 \%$ paraformaldehyde. For immunofluorescence or pre-embedding immunogold labeling, samples are treated with $0.5 \%$ Triton X-100. The detergent will extract membranes. The sample is then post-fixed with $2 \%$ glutaraldehyde before resin embedding.

The contrast in the low magnification image (Figure 1A) is proportional to the mass density of the specimen. At this magnification and position in the energy loss spectrum $(155 \mathrm{eV})$ only the condensed chromatin at the nuclear envelope and the well-contrasted nucleoli are clearly discerned. By recording images before and after the phosphorus ionization edge ( $\mathrm{L}_{\text {II,III }}, 132 \mathrm{eV}$ ) (Bazett-Jones \& Hendzel 1999), a net phosphorus image is obtained through dividing the post-edge image, recorded at $155 \mathrm{eV}$, by a pre-edge image, recorded at $120 \mathrm{eV}$. In the net phosphorus image (Figure 1B), blocks of condensed chromatin, primarily along the nuclear periphery but also sparsely dispersed throughout the nucleoplasm, are evident. Less-condensed chromatin is unevenly distributed throughout the nucleoplasm. The nitrogen map (Figure 1C), created by the ratio image of a post nitrogen edge $(415 \mathrm{eV})$ and a prenitrogen edge $(385 \mathrm{eV})$ image, contrasts both nucleic acid and protein, and as a result contrasts additional structures not detected in the phosphorus distribution. Differences between the phosphorus and nitrogen maps of the nucleolus are more striking. The nitrogen distribution of the nucleolus is relatively uniform, whereas regions of high and low phosphorus content are apparent. Relationships between phosphorus-rich, nucleic acid-rich structures and protein structure depleted in nucleic acid are readily seen in overlays of the phosphorus and nitrogen maps using computergenerated color scales (Figure 1D). Structures such as nuclear pores are rich in nitrogen only (green); chromatin is rich in phosphorus (yellow); and nucleoplasmic structures and the bulk of the nucleolus are intermediate in the ratio of phosphorus and nitrogen. Another approach to visualize the relationships of nucleic acid structures vs. protein structures is illustrated in Figure 1E,F. By subtracting the phosphorus map from the nitrogen map, normalized over regions of the specimen that contain neither (resin outside of the cell), protein-based structures that are not rich in nucleic acid become well contrasted (Figure 1E). If the phosphorus signal is then super- 

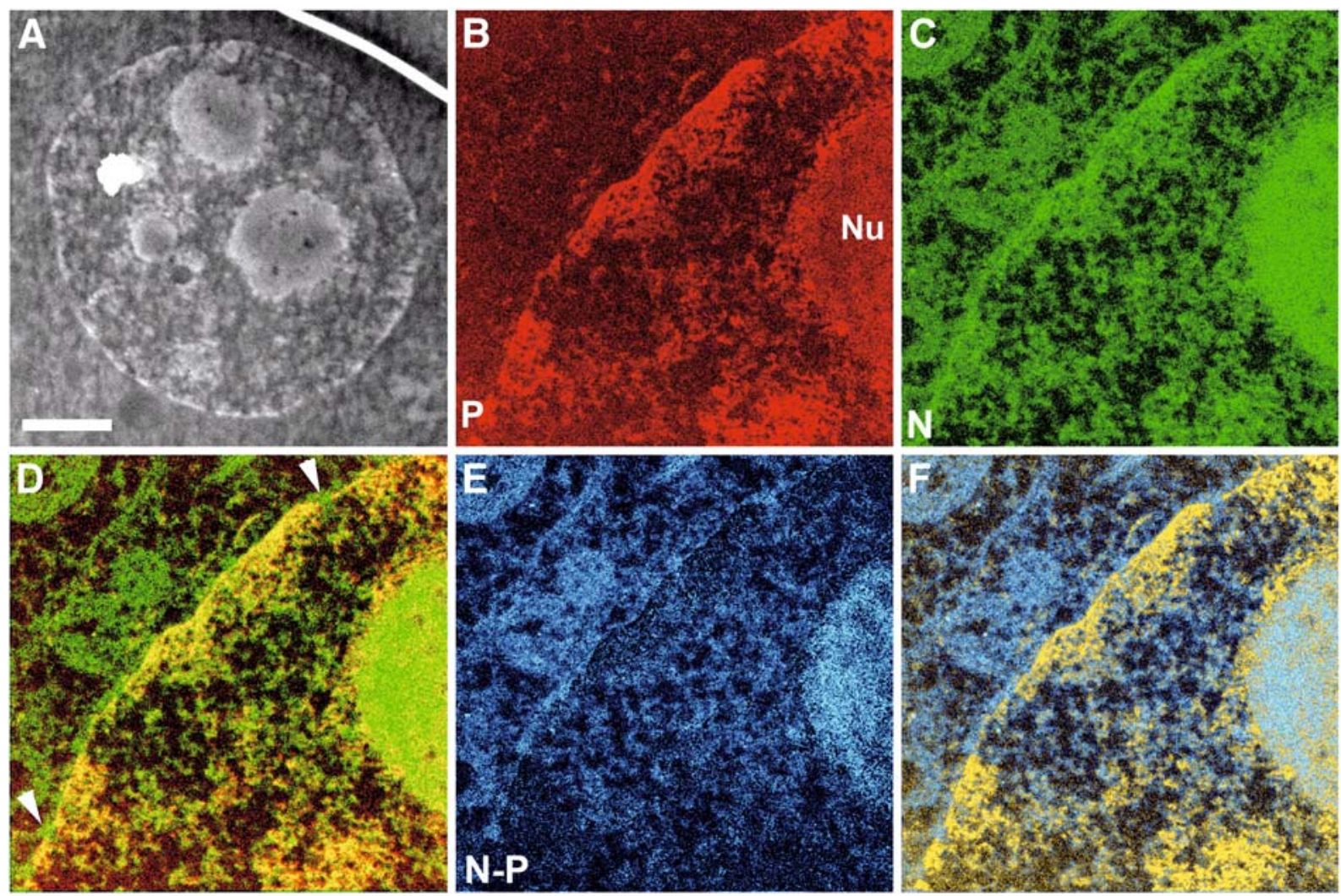

Figure 1. Electron-spectroscopic images of a nucleus from a 16-cell stage pre-implantation mouse embryo. (A) Mass-sensitive image of nucleus, recorded at $155 \mathrm{eV}$. (B) Phosphorus map (P) of a region of the nucleus shown in (A), generated by calculating the ratio of a phosphorus post-edge image $(155 \mathrm{eV})$ to a pre-edge image $(120 \mathrm{eV})$. (C) Nitrogen map $(\mathrm{N})$ of the same field shown in (B), generated by calculating the ratio of a nitrogen post-edge image $(415 \mathrm{eV})$ to a pre-edge image $(385 \mathrm{eV})$. (D) Merged image from those shown in (B) and (C). (E) A nitrogen-phosphorus map (N-P), calculated by subtracting the phosphorus map from the nitrogen map, after alignment and normalization over a background region containing neither phosphorus nor nitrogen (resin outside of embryo boundary). (F) Phosphorus map (P) (yellow) merged with nitrogen-phosphorus (N-P) map. Scale bar represents $1.5 \mu \mathrm{m}$ in (A), $500 \mathrm{~nm}$ in (B-F).

imposed on the N-P image, chromatin's relationship to protein structures near to, but not overlapping, the chromatin are apparent. For instance, the nitrogenrich nuclear pore complexes, which are intimately associated with chromatin on the nuclear periphery, can be distinguished from the chromatin.

The advantage that ESI provides over CTEM in imaging the cell nucleus is illustrated in Figure 2. Two serial sections of a cell were prepared. One section was stained with uranyl acetate and lead citrate, whereas the second serial section was not stained. Both sections were stabilized by coating with a $3 \mathrm{~nm}$ thick carbon film as described previously (Dellaire \& Bazett-Jones 2004). An unstained serial section was required to create the phosphorus and nitrogen maps because the heavy metals interfere with elementspecific mapping. A low-magnification bright-field image of the stained section (Figure 2A) and the same image with its contrast inverted (Figure 2A,B) reveal uniform contrast throughout the nucleus, with only a slight increase in contrast along the nuclear envelope. The mass-sensitive, energy loss dark-field image of the unstained section (Figure 2C) also demonstrates a high mass density along the nuclear periphery, corresponding primarily to condensed chromatin, but displays a lower contrast of material in the nuclear interior than that seen in the stained section imaged in bright-field mode. This illustrates that the binding of the heavy atoms is not necessarily proportional to biological mass density. (The term 'electron-dense' is frequently used in the literature to refer structures imaged by heavy-atom staining and bright-field CTEM. This term should always be replaced with 'stain-rich', since the biological 

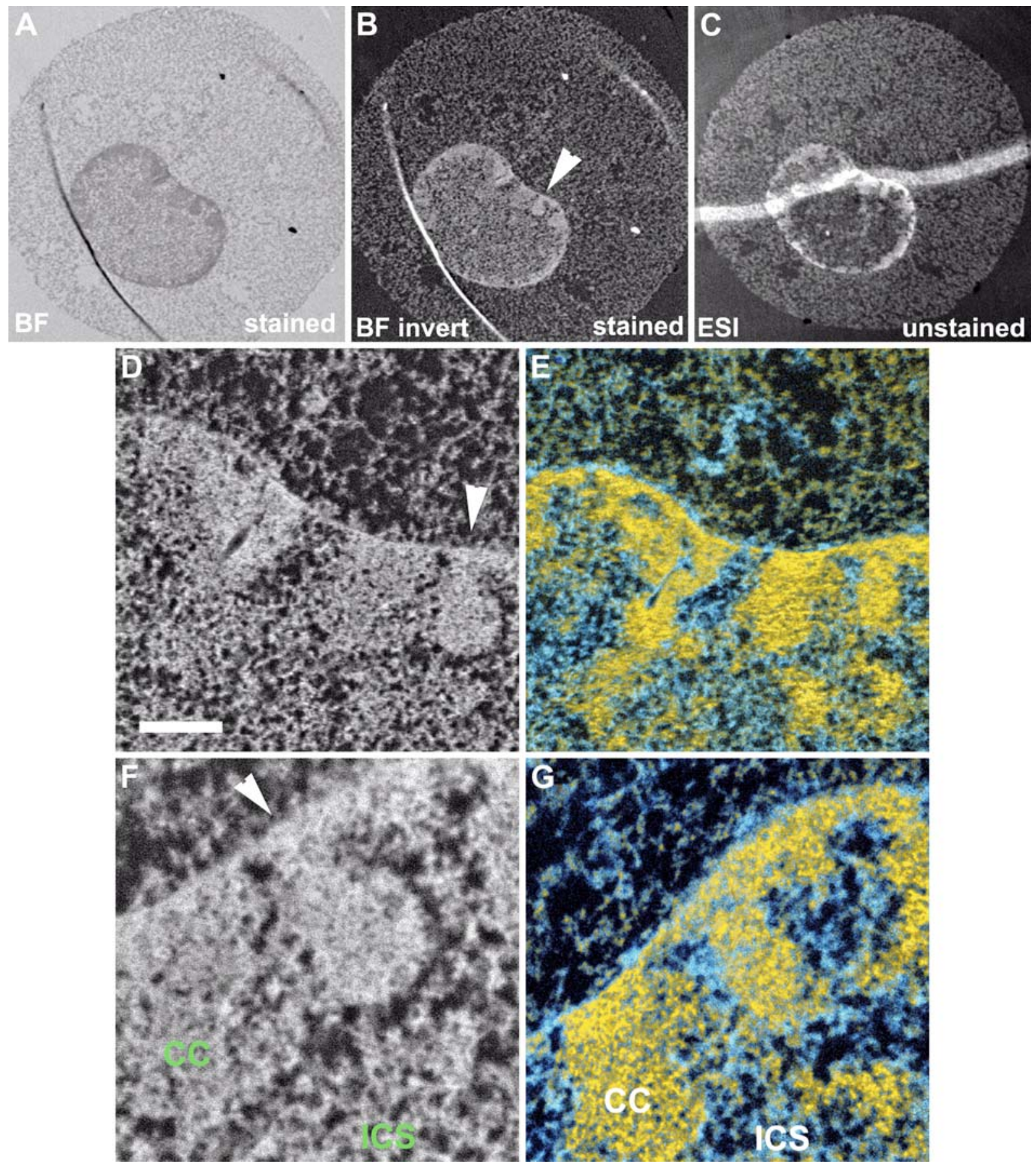

Figure 2. Comparisons of serial sections imaged with stain and bright field vs. no stain and ESI. (A) Section of a mouse erythroleukemia cell stained with $2 \%$ uranyl acetate, $0.2 \%$ lead citrate, imaged in bright field. (B) Image in (A) shown with inverted contrast. (C) ESI of a serial section from that shown in (A). This section was not stained, and was imaged at $155 \mathrm{eV}$, providing a mass-sensitive, energy-loss dark-field image. The white band indicates where the stabilizing carbon film has folded over itself. This thin carbon film does not contribute density to the net elemental signals. (D) Higher-magnification image of a field from the same nucleus as in (A). The arrowheads provide a fiduciary reference. (E) Higher magnification of a field from the nucleus shown in (C). The nitrogen-phosphorus map (blue) is superimposed with the phosphorus map (yellow). (F, G) Higher-magnification images of fields shown in (D) and (E), respectively. Condensed chromatin (CC) and interchromosome space (ICS) are indicated. Scale bar represents $2.6 \mu \mathrm{m}$ in (A-C), $500 \mathrm{~nm}$ in (D, E) and $25 \mathrm{~nm}$ in (F, G). 
structure may itself have a high affinity for the stain but not be electron dense.) In the higher magnification views of the bright-field images (Figure 2D,F), it is not possible to distinguish between condensed chromatin and other types of material, such as RNPs or decondensed chromatin associating with proteinbased complexes. For example, there is little density modulation or morphological information to distinguish between the condensed chromatin (CC) and the material found in the interchromosome space (ICS). These two subdomains, however, can easily be distinguished by the phosphorus and nitrogen mapping in the adjacent unstained section (Figure 2E,G).

\section{Visualizing subdomains of the multifunctional nucleolus with ESI}

The nucleolus has been identified as the subnuclear 'organelle' that is responsible for ribosome biogenesis (Vincent \& Miller 1966). This process in mammals begins with clustering and transcription of ribosomal RNA genes from a number of chromosomes. The transcripts are processed and, together with numerous large and small subunit proteins, preribosomes are assembled and stored. However, more recently it has become apparent that the nucleolus may serve other functions, unrelated to ribosome biogenesis (Pederson \& Politz 2000, Leung \& Lamond 2003). These functions include the maturation of non-nucleolar RNAs, mRNA export, regulation of cell senescence, control of viral infection, cell cycle progression, tumor suppression, and DNA repair. High-resolution structural studies will be required to understand how such a large variety of seemingly unrelated processes can occur simultaneously on the same platform. ESI may play in a role in such studies. ESI results to date provide both confirmatory and new, higher-resolution information that refines and extends the current model of nucleolar organization.

The nucleolus may be thought to be a dense subnuclear organelle that would limit the diffusion of macromolecules into its interior, such as p53, Mdm2, p14/19ARF, Rb, ING1, etc. (Dellaire \& Bazett-Jones 2007). The ESI data shown in Figure 3A,B shows that the interior is not necessarily a uniformly dense structure, and demonstrates the potential for proteins such as Mdm2 or ARF to diffuse into the nucleolar interior to associate in complexes either for storage or in signal transduction processes. The mass-sensitive image shows that numerous channels comprise the interior through which molecules could transit. Measurements of the mass density of the nucleolus suggest that it is approximately twice as mass dense as the surrounding nucleoplasm. The ratio of the mass density in the nucleolus compared to the nucleoplasm in the region indicated by the box in Figure $3 \mathrm{~A}$ is $1.93(112 / 58)$. A 2-fold difference in mass density may reflect a more crowded environment but would not necessarily preclude a high diffusion constant (Grünwald et al. 2008). The opportunities to corral molecules may in fact be fewer in the nucleolar interior due to the lower concentration of chromatin fibers. Even outside of the channels, the large proteinfilled spaces between the granules of the granular component indicate that proteins would be able to traffic relatively unhindered through these subregions of the nucleolus. It may be in these spaces between the pre-ribosomes that the molecules that participate in non-ribosome biogenesis events reside and interact.

The ESI data also show that a major component of the fibrillar center is DNA (nucleolar DNA in Figure 3E,F, and arrowhead in Figure 3G). Quantification of phosphorus and nitrogen levels also reveals biochemical relationships of protein and nucleic acid composition in subnucleolar compartments. Comparisons of $\mathrm{P}$ and $\mathrm{N}$ ratios can be converted to stoichiometric relationships using internal standards such as chromatin or ribosomes (Bazett-Jones et al. 1999). (This approach is superior to quantification from electron energy loss spectra. Reliable values for the partial cross-section of scattering of these elements, required for quantification from spectra, have not been determined.) The P:N ratio of chromatin

Figure 3. ESI of an SK-N-SH (human neuroblastoma) cell nucleus. (A, B) Low-magnification mass-sensitive images. The ratio of mass density of the region corresponding to the nucleolus, relative to the nucleoplasm (box in A) is 1.93 (112/58). (C) Phosphorus map of a field from (B) at higher magnification. Arrowheads provide a fiduciary reference. (D) Nitrogen-phosphorus (N-P) map (blue) merged with phosphorus map (P) (yellow). Granular component (GC), dense fibrillar component (DFC) and chromatin (Ch) are indicated. (E, F) Higher-magnification of a field from (D). Chromatin in the nucleoplasm and chromatin within the nucleolus, possibly corresponding to rDNA genes (NuChromatin), and the same structures as in (D), are indicated in (E). Arrowheads denote channels through the interior of the nucleolus. (G) Merged N-P (blue) and $\mathrm{P}$ map (yellow) of a nucleolus from a different cell from those in (A-F). NuCh refers to nucleolar chromatin in a fibrillar center. Scale bar represents $4 \mu \mathrm{m}$ in (A, B), $710 \mathrm{~nm}$ in (C, D), 330 $\mathrm{nm}$ in $(\mathrm{F})$, and $115 \mathrm{~nm}$ in $(\mathrm{G}) .(\mathrm{A}-\mathrm{F})$ adapted from Grünwald et al. (2008); (G) adapted from Politz et al. (2005). 

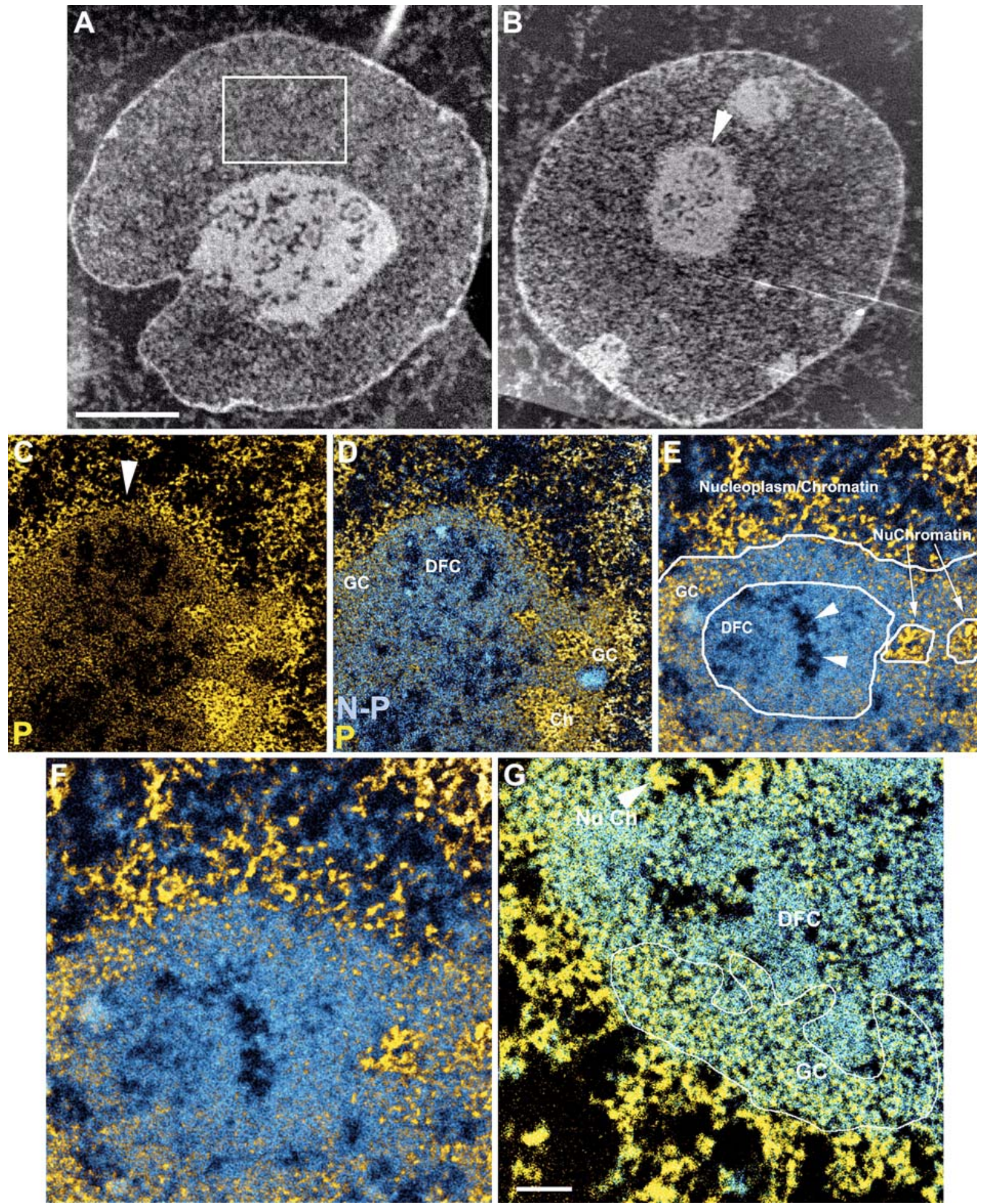
(measured from the nucleus represented in Figure 3G is 0.129 (based on nucleosomal composition), a value similar to the measured $\mathrm{P}: \mathrm{N}$ ratio of the ribosomal gene chromatin in the fibrillar center (0.140) (refer to figure 5 in Politz et al. 2005). The P:N ratio over large regions of either the dense fibrillar component (0.079) or the granular component $(0.081)$ predicts an overall nucleic acid content of $31 \%$. However, the $\mathrm{P}: \mathrm{N}$ ratio of the granules themselves in the granular component (0.116) is significantly higher than that of the overall granular compartment, and predicts a $45 \%$ nucleic acid content of the granules. This nucleic acid content is similar to that of mature ribosomes (54\%). The difference in the P:N content of the granules in comparison with that of the entire granular component predicts that $14 \%$ of the granular component, corresponding to the spaces between the granules, is composed of protein, and that this intergranule protein is not co-resident with nucleic acid. This is further supported qualitatively by the even larger spaces between the granules in the granular component seen in Figure 2F. We conclude that this ribosome-free domain of the granular component is populated by macromolecules that likely serve other functions.

\section{Distinguishing RNA and DNA by phosphorus and nitrogen mapping}

In mammalian cells, interchromatin granule clusters (IGCs), also referred to as speckles, are subnuclear compartments containing components of the pre-mRNA splicing machinery. They are rich in the serine-arginine (SR) family of proteins, and have been characterized by immunofluorescence with the antibody against the conserved splicing factor, SC35 (Fu \& Maniatis 1990; Mintz et al. 1999), providing the 'speckled' distribution pattern. IGCs are also rich in pre-mRNA processing factors such as snRNPs and hnRNPS, transcription factors and numerous other proteins (Mintz et al. 1999).

The interior of IGCs is devoid of DNA (Thiry 1993), but chromatin on the periphery of these compartments is highly dynamically acetylated, a marker for transcriptionally active chromatin (Hendzel et al. 1998). Additionally, gene-rich chromosome bands have been shown to cluster around SC35 domains, indicative of euchromatin and transcriptionally active neighborhoods where IGCs serve as functional centers (Shopland et al. 2003). IGCs likely represent storage, assembly and modification sites for splicing factors.

In interphase cells, IGCs are generally $1-2 \mu \mathrm{m}$ in diameter. ESI reveals that they are composed of phosphorus-rich granules that range from 5 to $20 \mathrm{~nm}$ in diameter (Figure 4) (Hendzel et al. 1998). The total mass and stoichiometric relationship between protein and RNA, based on the phosphorus and nitrogen maps, has been calculated to show that the granules contain hnRNA (Hendzel 1998). The granules are embedded within a network of protein fibers, which also form contacts with the surrounding chromatin (Figure 4). Since the spectral analysis performed by ESI alone cannot distinguish between phosphorus contributed by DNA or RNA, other criteria are required to delineate DNA-based structures from RNP structures. As seen previously in the discussion of the nucleolus, the RNP-based structures, such as pre-ribosomes, have a phosphorus-tonitrogen ratio that can be distinguished from that of chromatin. Because of this, the granular component can easily be distinguished from the surrounding chromatin by comparing the phosphorus and nitrogen maps. The same principle applies to IGCs. Again, the nitrogen to phosphorus ratio is significantly higher in the core of the IGC compared to that of the surrounding blocks of chromatin. In addition, the fine 'granular' nature contributed by the RNP granules, allows the RNP to be distinguished from chromatin by taking into account this morphological difference. Using the criteria of a higher nitrogen to phosphorus ratio and the granularity of RNPs, RNPs can

Figure 4. ESI of an SK-N-SH human neuroblastoma nucleus. (A) Low-magnification mass-sensitive image $(155 \mathrm{eV})$. (B) Overlay of the low-magnification mass image on a fluorescence image recorded from the same physical section. SC35 was immunofluorescently labeled with a $\mathrm{Cy} 3$ secondary antibody. Interchromatin granule clusters (IGC) could be identified for ultrastructure analysis by correlative fluorescence microscopy and ESI. (C) Phosphorus map (P) of a region of the nucleus in (A). (D) Nitrogen-phosphorus map of the region shown in (C). (E) Merged nitrogen-phosphorus map (blue) and phosphorus map (yellow). (F) Higher magnification of the field shown in (E). Silver-enhancement of a nanogoldtagged secondary antibody directed against the same primary against SC35 (as in B) reveals the SC35 distribution. The silver detection is enhanced due to an energy loss peak that is detected with the nitrogen signal $(415 \mathrm{eV} / 385 \mathrm{eV})$. Arrowhead denotes RNP granules in the IGC, and arrow denotes chromatin fibers at the periphery of the IGC. Scale bar represents $2 \mu \mathrm{m}$ in (A, B), $440 \mathrm{~nm}$ in $(\mathrm{C}-\mathrm{E})$, and $230 \mathrm{~nm}$ in $(\mathrm{F})$. 

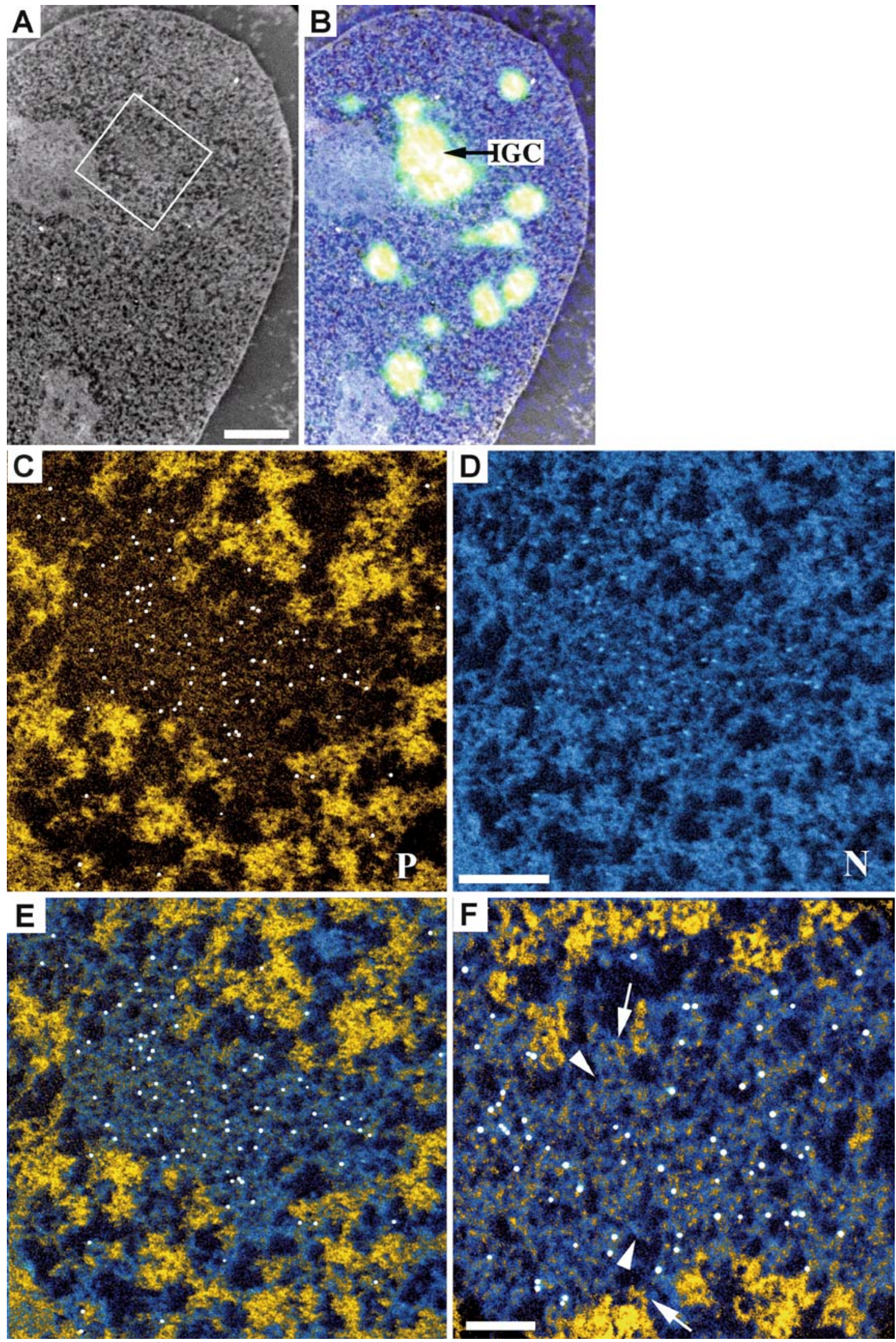

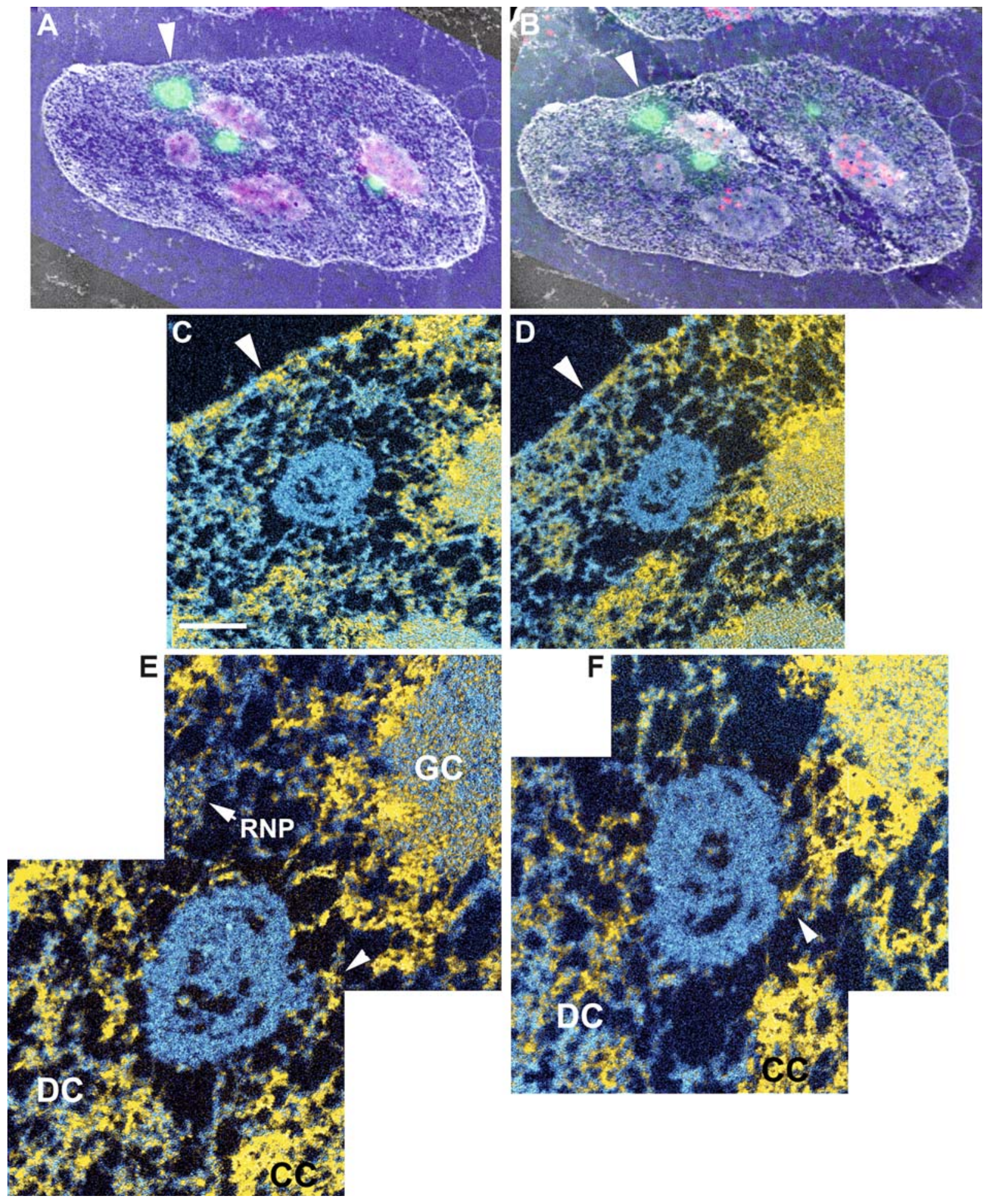
Figure 5. Correlative fluorescence microscopy and ESI of a mouse embryonic stem cell nucleus. (A, B) Serial physical sections of immunofluorescently labeled cells were first imaged with the fluorescence microscope. The two physical sections were then imaged at low magnification to generate a mass-sensitive image by ESI. Merging the two images permitted the identification of PML NBs in the nuclei. (C, D) A PML NB was imaged in each serial physical section, by generating both phosphorus and nitrogen maps. The nitrogen-phosphorus maps (blue) are merged with the phosphorus maps (yellow). Arrowheads serve as fiduciary markers to relate the low-magnification views from (A, B). (E, F) Highermagnification views of the PML NB from $(\mathrm{C}, \mathrm{D})$. Condensed chromatin (CC) and the granular component (GC) of the nucleolus are near the PML NB. Decondensed chromatin (DC) surrounds the body and appears to make contact with the protein-based core of the PML NB. A cluster of RNP granules is also found near the body. Scale bar represents $2 \mu \mathrm{m}$ in (A, B), $0.5 \mu \mathrm{m}$ in $(\mathrm{C}, \mathrm{D})$, and $260 \mathrm{~nm}$ in $(\mathrm{E}, \mathrm{F})$.

frequently be identified in the vicinity of $10 \mathrm{~nm}$ chromatin fibers (Figure 5F, Figure $6 \mathrm{H}$ ) even when they are not concentrated into easily recognized subregions such as IGCs.

\section{ESI provides clues to the functional role of PML nuclear bodies}

The promyelocytic leukemia nuclear body (PML NB) can be described as a subnuclear 'organelle', which we think plays a major role in chromatin organization and serves to compartmentalize several nuclear functions (Dellaire \& Bazett-Jones 2004). Although PML NBs are implicated in regulating many nuclear activities, it is still unclear how they function. A prevailing model is that they serve as storage sites of nuclear proteins. Another model proposes that they act as platforms where multi-subunit macromolecular complexes accumulate and where posttranslational modifications of regulatory molecules take place, p53 being a prime example (Dellaire \& Bazett-Jones 2004). ESI and analysis of the biochemical composition and dynamics of PML NBs together provide clues that support another model. Although this revised model does not exclude the storage or platform concepts, we propose that PML NBs interact directly with the surrounding chromatin, and thereby perform a more active role in servicing the regulation of genes on their periphery. Several findings support this model.

First, all of our images of PML NBs acquired with ESI clearly indicate that the core of the PML NB is a protein-based structure that is completely devoid of either RNA or DNA (Boisvert et al. 2000, Eskiw et al. 2003, Dellaire \& Bazett-Jones 2004). The images, however, provide compelling support for physical contacts between the periphery of the protein core and the surrounding chromatin fibers (Figure 5C-F). We have argued that these contacts are functional since the positional stability of PML NBs is dependent on chromatin contacts (Eskiw et al. 2004). In previous studies, we have also shown that the structural integrity of PML NBs is severely affected by DNA damage (Eskiw et al. 2004, Dellaire et al. 2006). Our findings indicate that PML NBs are exquisite sensors of DNA damage and other cellular stresses.

Second, since PML NB integrity is dependent on chromatin contacts, we hypothesized that PML NBs may be destabilized as these contacts are effected through changes in topological constraints during DNA replication in S-phase. Indeed, we showed that PML NBs break down by a fission mechanism during the replication of euchromatin in early S-phase, when observed in live cells with fluorescence microscopy. Importantly, PML NB number increases approximately two-fold in some cell types during this time period (Dellaire et al. 2006). We hypothesize that topological changes in replicating chromatin domains bound to PML NBs cause disruption of the bodies (Dellaire et al. 2006). ESI also revealed a dramatic structural instability in PML NBs during early S-phase. The protein cores of the bodies were completely disrupted, leading to the redistribution of PML protein along the chromatin fibers.

The theme that emerges from all of these preliminary studies, in which ESI provided the initial concept, is that a direct and active relationship exists between PML NBs and chromatin. Since PML NB instability and duplication occur in early S-phase, the bodies may have a stronger association with euchromatin than heterochromatin, since euchromatin is replicated in early $\mathrm{S}$-phase. This is a radically different concept from that of PML NBs serving only as passive storage sites or accumulations of multiprotein complexes. Instead, ESI has contributed to the hypothesis that PML NBs interact with specific chromatin loci and contribute to the transcriptional regulation of these associated genes. This concept is currently being tested with complementary approaches.

Serial sections of PML NBs and the surrounding nucleoplasm reveal other features that may be relevant to their role (Figure 5). One feature is the 

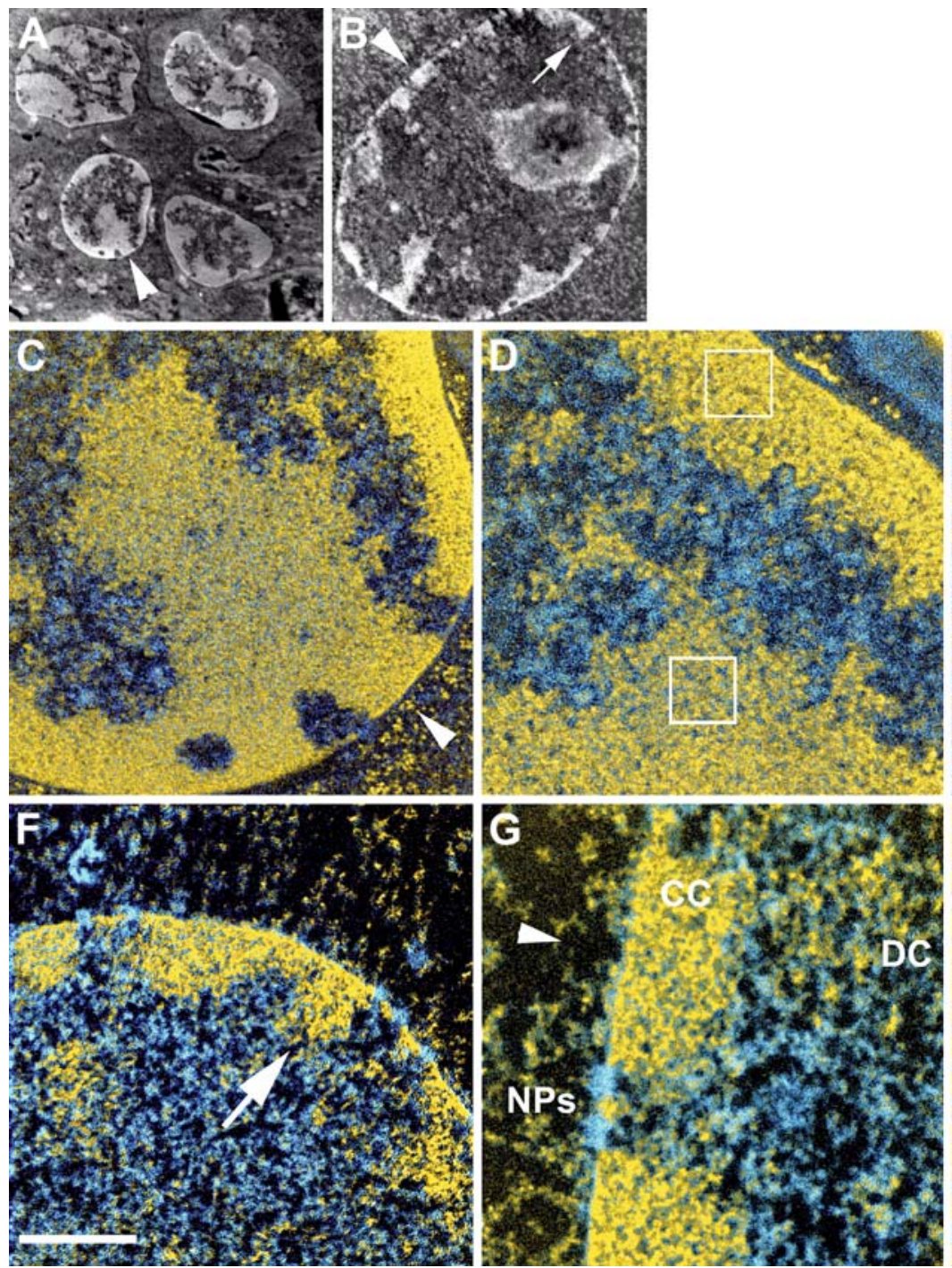
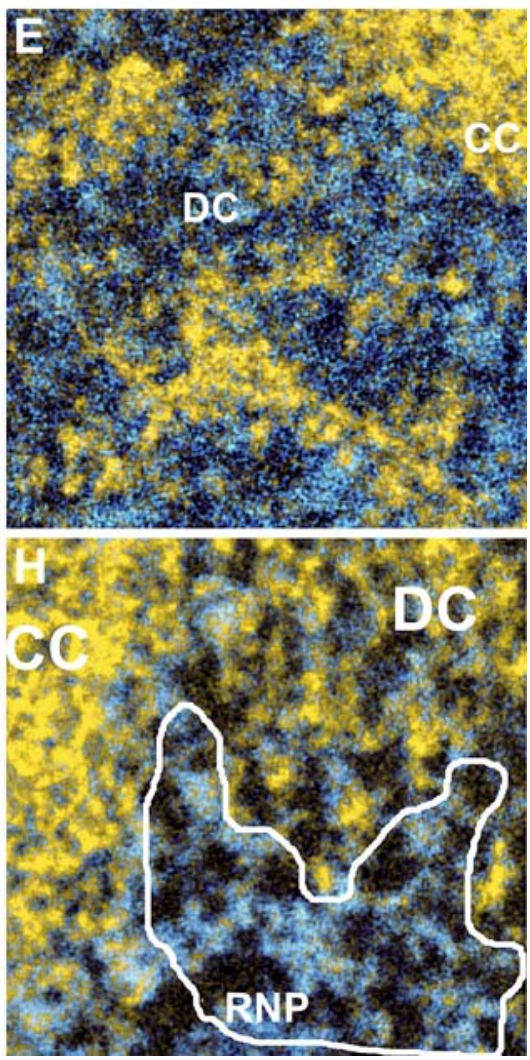

Figure 6. ESI of a lymphocyte and a mouse erythroleukemia cell (MEL) nucleus. (A, B) Mass-sensitive images (155 eV) of a lymphocyte nucleus from a mouse spleen, and an MEL cell nucleus, respectively. (C, D) Higher-magnification images of the lymphocyte nucleus. Arrowheads serve as fiduciary markers between the low- and higher-magnification images. Merged nitrogen-phosphorus maps (blue) and phosphorus maps (yellow) can be used to distinguish chromatin fibers from the nucleoplasmic protein-based structures, and to visualize the qualitative differences in fiber density in different regions of condensed chromatin (boxes in D). (E) Higher-magnification view of a region from (D). Condensed chromatin (CC) and a region of decondensed chromatin (DC) between the condensed chromatin domains are presented. $(\mathbf{F}-\mathbf{H})$ Similar presentation as in (B-D), but for the MEL cell nucleus. The higher magnification images in $(\mathrm{G}, \mathrm{H})$ are of a different region from that shown in (F). The two regions are indicated in (B). Nuclear pores (NP) are indicated in (G), and dispersed RNP granules are indicated adjacent to the decondensed chromatin in (H). Scale bar represents $4.2 \mu \mathrm{m}$ in (A, B); $1 \mu \mathrm{m}$ in $(\mathrm{C}, \mathrm{F}) ; 500 \mathrm{~nm}$ in $(\mathrm{D}, \mathrm{G}) ; 250 \mathrm{~nm}$ in (E, H).

structure of the protein core of the body itself. In this undifferentiated murine embryonic stem (ES) cell, the core appears porous, with gaps or holes between layers of protein. The structure of the protein core of bodies varies from one cell type to another. In osteosarcoma cells (U2OS) over-expressing the PML IV alternatively spliced isoform, for example, the protein core consists of fibers (unpublished data), whereas in the same cells over-expressing the PML I splice isoform, the core of the bodies consists of a 
more densely packed protein matrix. These structural differences may reflect the way these various splice isoforms of PML protein assemble to create the body, or may reflect a different composition of the other proteins that are known to accumulate in the body, such as Sp100, CBP or Daxx, as examples. Correlation of the structure, composition, and function of PML NBs from one cell type to another awaits further investigation.

The contacts between PML NBs and the surrounding chromatin may be related to a transcriptional regulatory role. PML NBs appear to locate in transcriptionally active domains of the cell nucleus at the level of resolution of the fluorescence microscope (Wang et al. 2004). This is supported by ESI in that RNP structures are routinely observed in the immediate vicinity of PML NBs (Figure 5E). A complete 3D analysis of the relationships of PML NBs with condensed chromatin, decondensed chromatin, and RNPs, through serial section reconstructions, and under conditions of specific physiological manipulations, such as transcriptional inhibition, may reveal the role of PML NBs in gene regulation.

\section{Degrees of chromatin compaction revealed by ESI}

Chromatin organization within the nucleus is nonrandom. One example of this is the discrete volume that is occupied by each chromosome, referred to as a chromatin territory. Their relative locations are nonrandom (Boyle et al. 2001, Chambeyron \& Bickmore 2004, Bolzer et al. 2005). Moreover, particular genes are sometimes found in the interior of their territories and sometimes at the periphery of the territories. Besides territory organization, chromatin is also organized according to a state of condensation or compaction. Chromatin density is typically higher along the nuclear envelope and around the nucleolus of many cell types. Much of this chromatin is late replicating and is defined as heterochromatin. Some heterochromatin is thought to be physically compacted in all cells, referred to as constitutive heterochromatin, whereas facultative heterochromatin represents silenced regions of the genome that vary from one cell type to another. All of these physical and biochemical properties of chromatin are thought to reflect epigenetic marks that distinguish one type of chromatin from another. These epigenetic features include methylation of the DNA bases themselves, and modifications of the histone molecules that are responsible for packaging DNA, modifications that include phosphorylation, acetylation, methylation, SUMOylation, and ubiquitination. Epigenetic regulators undoubtedly play a critical role in cellular differentiation and development. These will affect the chromatin landscape, which can be extensively characterized by a variety of microscopical techniques.

The opportunities offered by ESI to visualize chromatin fibers at the $10 \mathrm{~nm}$ level of organization, and to distinguish chromatin from the surrounding protein-based components of the cell nucleus, may contribute to a more detailed understanding of this chromatin landscape. Some of the structural features of chromatin that can be addressed by ESI are illustrated in Figure 6. First, the relative volume of the nucleus that is occupied by highly condensed chromatin can be unambiguously determined by phosphorus and nitrogen mapping. Major differences can be qualitatively described in the two examples shown here of a lymphocyte imaged in the mouse spleen (Figure 6A,C-E) and of a cultured murine erythroleukemia (MEL) cell (Figure 6B,F-H). The volume occupied by nucleoplasmic space is cell type-dependent, ranging from approximately $40 \%$ to as high as $80 \%$ (unpublished data). The ratio of decondensed chromatin to condensed chromatin also varies dramatically from one cell type to another. Both cell types shown here have significant accumulations of chromatin along their nuclear periphery, though much more in the case of the lymphocyte. Some cells, however, such as cultured fibroblasts have only a thin rim of condensed chromatin at the periphery. This illustrates that a what is classed as constitutive heterochromatin based on biochemical markers may not correlate with what is considered heterochromatin based on structural features. Future studies should be directed at correlating biochemical markers of facultative and constitutive heterochromatin with the actual structure of these chromatin regions.

ESI also reveals differences in condensation of DNA fibers even within the peripheral heterochromatin domains themselves. In the example shown in Figure 6C,D, the chromatin at the immediate periphery (upper box in Figure 6D) is more densely packed than that towards the interior (lower box in Figure $6 \mathrm{D})$. The more interior region, delineated by one of the boxes, shows protein-based components between the chromatin fibers (blue signal), which are absent in the peripheral condensed chromatin. In addition, 
the degree of packing of the chromatin fibers at the immediate periphery of the MEL cell nucleus is noticeably less than that in the peripheral chromatin of the lymphocyte. In the MEL cell nucleus, $10 \mathrm{~nm}$ chromatin fibers can occasionally be identified in this peripheral chromatin (Figure $6 \mathrm{H}$ ). Such individual $10 \mathrm{~nm}$ fibers cannot be distinguished in the condensed chromatin at the periphery of the lymphocyte nucleus. The biochemical basis for these differences must be linked to the epigenetic markers. Correlation of the biochemical markers with the ultrastructural differences awaits further analysis.

Besides the highly condensed chromatin along the nuclear periphery, the interior of the nucleus in both cell types consists of dispersed, decondensed $10 \mathrm{~nm}$ chromatin fibers. These fibers are separated by nucleoplasmic space, consisting of protein that resisted extraction, and clusters of RNPs (Figure 6G,H). In some cell types, most of the chromatin consists of decondensed $10 \mathrm{~nm}$ fibers. These fibers form a meshwork that is interspersed with RNP clusters and nuclear bodies (Dehghani et al. 2005). In other cell types, such as the lymphocyte (Figure 6A,C-E), most of the chromatin is highly condensed with only a relatively small fraction represented as a mesh of 10 $\mathrm{nm}$ fibers. The MEL cell (Figure 6B,F-H) represents an intermediate between highly condensed and highly extended chromatin states.

\section{Conclusions and prospects}

The theme of compartmentalization of functions within the nucleus has emerged over the past few years, transforming the way we think of the regulation of nuclear processes. Imaging approaches, over conventional biochemistry, have been responsible for this revolution. Clearly, improvements of existing technologies and the advent of new ones will continue to inform our models of nuclear organization and function. Immunofluorescence microscopy has been a central player by identifying the substructures or nuclear 'organelles', such as Cajal bodies or PML nuclear bodies. Its advantage is the minimal effort required in sample preparation, and the large numbers of cells that can be studied, combining the advantages of describing characteristics of single cells with statistical analysis of subpopulations within large numbers of cells. Fluorescent tagging of proteins, with molecules such as GFP, has elevated our concepts of nuclear function by providing information on the dynamic behavior of molecules in living cells. These fluorescent microscopical methods have recently benefited from technological innovations that are dramatically improving both lateral and axial spatial resolution, some of these developments being featured in this issue. Regardless, electron microscopy will remain the 'gold standard' for spatial resolution for some time, in spite of the loss of dynamic information due to the preparative steps required.

In this review, we have demonstrated advantages of an analytical electron microscopy method. ESI requires only the same fixation and preparative steps required for conventional electron microscopy, but provides higher contrast and biochemical detail at the macromolecular level of spatial resolution. Specifically, structures and supramolecular structures that are based on either protein, DNA or RNA can be distinguished. We anticipate that ESI will play a significant role in determining the structural consequences on chromatin compaction and condensation of the increasing list of epigenetic modifications of chromatin. Elucidating these changes in cellular differentiation will have profound functional consequences.

The next steps in applying this technology are to combine it with other standard approaches used in electron microscopy. One of these is serial sectioning. Complete volumes of chromosome territories or nuclear bodies are required to understand the heterogeneity in function within these structures themselves. Three-dimensional information within single physical sections can also be extracted through tomographic methods. Tomography and ESI together would in principle be useful for investigating a number of subnuclear features; for example, for visualizing chromatin fiber interactions, for imaging protein-filled channels within chromosome domains or territories, and for extracting topological information on fiber arrangements and interactions of chromatin fibers with the surfaces of nuclear bodies such as the nucleolus, Cajal bodies, or PML nuclear bodies. The threedimensional information extracted from a single physical section could subsequently be added to that from consecutive serial sections, providing highresolution detail in large volumes within the nucleus.

Another area for future developments in ESI technology is in generating new metal tags for highresolution detection of specific proteins. This review has restricted discussion of element-specific mapping to phosphorus and nitrogen. But most of the 
lighter elements and some heavy elements in the periodic table are well suited for detection and mapping by ESI. Hence, expression of a metal-binding polypeptide fused to a protein of interest could be applied to mapping that protein, without the use of immunological reagents. Alternatively, such new metal-tagged fusion proteins could be detected simultaneously with both pre- and post-embedding detection using antibodies, facilitating the detection and mapping of multiple proteins in the same specimen. Besides the standard nanogold or colloidal gold reagents coupled to antibodies, streptavidin or other coupling intermediates to nanoparticles, such as quantum dots, can also be applied (Nisman et al. 2004) for detecting multiple proteins simultaneously. Even very small nanoparticles, which would not be detected in conventional electron microscopy, could be detected with ESI on the basis of element-specific contrast (e.g. cadmium) (Nisman et al. 2004).

\section{Acknowledgements}

We thank Reagan Ching and Dr Kashif Ahmed for their helpful comments on this manuscript. We thank Dr James Ellis for supplying the mouse erythroleukemia cell line. This work was supported by operating grants from the Canadian Institutes of Health Research, and the Natural Sciences and Engineering Research Council of Canada. D.P.B.-J. holds the Canada Research Chair in Cell and Molecular Imaging.

\section{References}

Bazett-Jones DP, Hendzel ML (1999) Electron spectroscopic imaging of chromatin. Methods 17(2): 188-200.

Bazett-Jones DP, Leblanc B, Herfort M, Moss T (1994) Shortrange DNA looping by the Xenopus HMG-box transcription factor, xUBF Science 264(5162): 1134-1137.

Bazett-Jones DP, Mendez E, Czarnota GJ, Ottensmeyer FP, Allfrey VG (1996) Visualization and analysis of unfolded nucleosomes associated with transcribing chromatin. Nucleic Acids Res 24(2): 321-329.

Bazett-Jones DP, Hendzel MJ, Kruhlak MJ (1999) Stoichiometric analysis of protein- and nucleic acid-based structures in the cell nucleus. Micron 30(2): 151-157.

Bazett-Jones DP, Kimura K, Hirano T (2002) Efficient supercoiling of DNA by a single condensin complex as revealed by electron spectroscopic imaging. Mol Cell 9(6): 1183-1190.

Boisvert FM, Hendzel MJ, Bazett-Jones DP (2000) Promyelocytic leukemia (PML) nuclear bodies are protein structures that do not accumulate RNA. J Cell Biol 148(2): 283-292.
Bolzer A, Kreth G, Solovei I et al. (2005) Three-dimensional maps of all chromosomes in human male fibroblast nuclei and prometaphase rosettes. PLoS Biol 3(5): e157.

Boyle S, Gilchrist S, Bridger JM, Mahy NJ, Ellis JA, Bickmore WA (2001) The spatial organization of human chromosomes within the nuclei of normal and emerin-mutant cells. Hum Mol Genet 10(3): 211-219.

Brown ML, Schroth GP, Gottesfeld JM, Bazett-Jones DP (1996) Protein and DNA requirements for the transcription factor IIIAinduced distortion of the $5 \mathrm{~S}$ rRNA gene promoter. $J$ Mol Biol 262(5): 600-614.

Chambeyron S, Bickmore WA (2004) Chromatin decondensation and nuclear reorganization of the HoxB locus upon induction of transcription. Genes Dev 18(10): 1119-1130.

Dehghani H, Dellaire G, Bazett-Jones DP (2005) Organization of chromatin in the interphase mammalian cell. Micron 36(2): 95-108.

Dellaire G, Bazett-Jones DP (2004) PML nuclear bodies: dynamic sensors of DNA damage and cellular stress. Bioessays 26(9): 963-977.

Dellaire G, Bazett-Jones DP (2007) Beyond repair foci: subnuclear domains and the cellular response to DNA damage. Cell Cycle 6(15): 1864-1872.

Dellaire G, Nisman R, Bazett-Jones DP (2004) Correlative light and electron spectroscopic imaging of chromatin in situ. Methods Enzymol 375: 456-478.

Dellaire G, Ching RW, Ahmed K et al. (2006) Promyelocytic leukemia nuclear bodies behave as DNA damage sensors whose response to DNA double-strand breaks is regulated by NBS1 and the kinases ATM, Chk2, and ATR. J Cell Biol 175(1): 55-66.

Eskiw CH, Dellaire G, Mymryk JS, Bazett-Jones DP (2003) Size, position and dynamic behavior of PML nuclear bodies following cell stress as a paradigm for supramolecular trafficking and assembly. J Cell Sci 116(Pt 21): 4455-4466.

Eskiw CH, Dellaire G, Bazett-Jones DP (2004) Chromatin contributes to structural integrity of promyelocytic leukemia bodies through a SUMO-1-independent mechanism. J Biol Chem 279(10): 9577-9585.

Fu XD, Maniatis T (1990) Factor required for mammalian spliceosome assembly is localized to discrete regions in the nucleus. Nature 343(6257): 437-441.

Grünwald D, Martin RM, Buschmann V et al. (2008) Probing intranuclear environments at the single molecule level. Biophys $J$ 94(7): 2847-2858.

Hendzel MJ, Boisvert F, Bazett-Jones DP (1999) Direct visualization of a protein nuclear architecture. Mol Biol Cell 10(6): 2051-2062.

Hendzel MJ, Kruhlak MJ, Bazett-Jones DP (1998) Organization of highly acetylated chromatin around sites of heterogeneous nuclear RNA accumulation. Mol Biol Cell 9(9): 2491-2507.

Kosak ST, Groudine M (2004) Gene order and dynamic domains. Science 306(5696): 644-647.

Leung AK, Lamond AI (2003) The dynamics of the nucleolus. Crit Rev Eukaryot Gene Expr 13(1): 39-54.

Mintz PJ, Patterson SD, Neuwald AF, Spahr CS, Spector DL (1999) Purification and biochemical characterization of interchromatin granule clusters. EMBO J 18(15): 4308-4320.

Misteli T (2001) Protein dynamics: implications for nuclear architecture and gene expression. Science 291(5505): 843-847. 
Nisman R, Dellaire G, Ren Y, Li R, Bazett-Jones DP (2004) Application of quantum dots as probes for correlative fluorescence, conventional, and energy-filtered transmission electron microscopy. J Histochem Cytochem 52(1): 13-18.

Osborne CS, Chakalova L, Brown KE et al. (2004) Active genes dynamically colocalize to shared sites of ongoing transcription. Nat Genet 36(10): 1065-1071.

Pederson T, Politz JC (2000) The nucleolus and the four ribonucleoproteins of translation. J Cell Biol 148(6): 1091-1095.

Politz JC, Polena I, Trask I, Bazett-Jones DP, Pederson T (2005) A nonribosomal landscape in the nucleolus revealed by the stem cell protein nucleostemin. Mol Biol Cell 16(7): 3401-3410.

Ren Y, Kruhlak MJ, Bazett-Jones DP (2003) Same serial section correlative light and energy-filtered transmission electron microscopy. J Histochem Cytochem 51(5): 605-612.
Shopland LS, Johnson CV, Byron M, McNeil J, Lawrence JB (2003) Clustering of multiple specific genes and gene-rich R-bands around SC-35 domains: evidence for local euchromatic neighborhoods. J Cell Biol 162(6): 981-990.

Stefanovsky VY, Pelletier G, Bazett-Jones DP, Crane-Robinson C, Moss T (2001) DNA looping in the RNA polymerase I enhancesome is the result of non-cooperative in-phase bending by two UBF molecules. Nucleic Acids Res 29(15): 3241-3247.

Thiry M (1993) Differential location of nucleic acids within interchromatin granule clusters. Eur J Cell Biol 62(2): 259-269.

Vincent WS, Miller OL Jr (1966) International Symposium on the Nucleolus. Its Structure and Function. J Natl Cancer Inst 23: 1-610.

Wang J, Shiels C, Sasieni P et al. (2004) Promyelocytic leukemia nuclear bodies associate with transcriptionally active genomic regions. J Cell Biol 164(4): 515-526. 